\title{
Stakeholders' Requirements Assessment for Biofuel Production
}

\author{
Mostafa F. Fawzy ${ }^{1}$, Paul J. Componation ${ }^{2}$, Guiping $\mathrm{Hu}^{3}$ \\ ${ }^{1,3}$ Industrial and Manufacturing Systems Engineering Department, Iowa State University, Ames, Iowa, USA \\ ${ }^{2}$ Industrial, Manufacturing, \& Systems Engineering Department, the University of Texas at Arlington, Arlington, Texas, USA
}

\begin{abstract}
Biofuel is one of the alternatives transportation fuel to substitute petroleum in the United States. Fast pyrolysis, as one of the most promising thermochemical based advanced biofuel production techniques, has been brought to the forefront of biofuel industry. With limited information about the manufacturing of fast pyrolysis units, a thorough understanding of stakeholders' requirements is timely and necessary. This study develops a comprehensive analysis of stakeholders' requirements. Subject matter experts were interviewed to rank, review, and evaluate a set of requirements for a variety of fast pyrolysis unit sizes. The requirements with the evaluations were then used to determine the most effective unit size. The analysis showed that the smallest unit size (process an average of 50 tons biomass per day) is the best alternative based on 50\% of the high-prioritized requirements. However, 37.5\% of the highprioritized requirements recommended the unit which process at least 2000 tons per day as the best alternative.
\end{abstract}

Keywords: Decision-making, Biofuel, Pyrolysis, Fast pyrolysis unit

\section{Introduction}

Renewable energy has been gaining attention globally. In the U.S., renewable energy production started in 1973 (EIA, 2001) and by 2011, $8 \%$ of the U.S. energy production came from renewable sources. This percentage increased to $9 \%$ in 2012 and then to $11.4 \%$ by 2013 (EIA, 2014). In 2013, 9.298 out of 81.669 quadrillion British thermal units came from renewable sources (EIA, 2014). Renewable energy has been used in transportation, industry, residence, commerce, and electric power consumptions (EIA, 2014). Approximately half of the renewable energy production is from biomass (EIA, 2014). Increasing attention has been attracted to biofuel production process.

Biofuel production has a large number of stakeholders. Each stakeholder has different perspective and requirements (Fawzy \& Componation, 2014). These different requirements can make the selection of a biofuel production strategy challenging. Therefore, optimizing the production of a Fast Pyrolysis Unit (FPU), is classified as a complex decision problem that can be solved using a Multi-Attribute Utility Theory (MAUT) approach (Min, 1994). The MAUT application follows the same pattern of normal decision-making approaches (Dyer, 2005). Decision makers often have to make decisions with limited information, especially in new areas such as advanced biofuel production. In order to design valid decisionmaking support systems to assist decision makers in these situations, a detailed analysis on the available information is required. This should also include investigation for factors that influence the decision under different conditions and situations. The current research explains the requirements-level analysis and its sensitivity analysis.

In this research, three alternatives FPUs sizes are analyzed. The first alternative is small FPUs with a capacity of 50 tons per day (tpd). The second alternative is a medium FPUs with a capacity between 200 to 500 tpd. Finally, the third alternative is the biggest FPUs with a capacity 2000 tpd or more.

A decision-making model was developed using Logical Decision software V7.2 to investigate the best FPUs' size based on stakeholders' requirement.

This study aims to investigate the impact of stakeholders requirements. This research also included a sensitivity analysis to determine the impact of high-prioritized requirements on FPU selection.

This research supports further development of an approach for the manufacturing, placement, and right sizing of FPUs for biofuel production infrastructure. Decision-makers face a lot of open-ended problems such as deciding FPUs size for biofuel production in real life. This problem is classified as an open-ended problem because it could have several correct answers based on different stakeholders' perspectives and requirements. Further, authors believe that the presence of additional information in the future may influence the decision and help better understand the problem. This may change the current results or decision based on the conceptual assessment of FPUs' sizes.

This research provides significant insights into complex decision problems with the use of powerful tool. In addition, the use of the Logical Decisions tool for the sensitivity and robustness analysis, as an integrated tool, developed better understanding of decision-making process. Moreover, this research presents an example of a decision-making approach that uses quantitative and qualitative criteria. However, a variety of methodologies, tools and techniques could be adopted to solve the problem. The suggested approach could be applied to other decision-making processes with limited information and multi-stakeholders involvement for selecting the best option from several alternatives.

This research provides an example of complex decisions that engineering managers are often faced where there are multiple stakeholders with sets of requirements. Engineering

\section{Volume 4 Issue 11, November 2015}




\section{International Journal of Science and Research (IJSR) \\ ISSN (Online): 2319-7064 \\ Index Copernicus Value (2013): 6.14 | Impact Factor (2014): 5.611}

managers could implement the proposed methodology for other complex problems analysis especially in renewable resources manufacturing. Engineering managers typically try to use a specific decision-making tool or set of tools to solve all decision problems (Componation \& Nicholls, 2011), but not all tools are suitable for every decision problem. This research supports the use of an integrated methodology for decision-making to solve decision problems without being restricted to only one tool.

\section{Literature Review}

FPU's size selection is considered as a complex decision problem due to a verity of stakeholders, multi-alternatives, and multi-requirements involved in the decision-making process. It is illustrated in the literature that there are multiple Biofuel Production Stakeholder (BPS) groups affecting the decision-making process each with a specific typology and perspective (Youngs, 2012; Fawzy \& Componation, 2014). Thirty-six groups are identified then classified into eight typology sets as BPS groups, which are involved in FPU's manufacturing (Fawzy \& Componation, 2014). That identification and classification of BPS groups was done by applying the theory of stakeholder identification, which was initiated by Freeman (1984).

Research studies published between 1996 and 2014 that mention biofuel manufacturing requirements were reviewed. In addition, a list of 31 requirements presented in a study done by Fawzy \& Componation (2015) as shown in Table 1 has been adopted as the base of the requirements recognition.

Table 1: References for Stakeholders' Requirements for Biofuel Production (Fawzy \& Componation, 2015)

\begin{tabular}{|c|c|c|c|c|c|c|c|c|c|c|c|c|c|c|c|c|c|}
\hline$\#$ & Requirement $\quad$ Year & $\stackrel{2}{2}$ & ஓे & ชิ & గి̊ి & ஓి & $\stackrel{\overbrace{}}{\check{\overbrace{}}}$ & ๖̊ & ঠे & 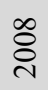 & ڤे & $\stackrel{\circ}{\stackrel{2}{\circ}}$ & ‡ & $\stackrel{\sim}{\stackrel{2}{2}}$ & $\frac{m}{2}$ & $\underset{⿱ 亠 凶}{\stackrel{\Xi}{\circ}}$ & $\stackrel{\text { त्ञ }}{\circ}$ \\
\hline 1 & $\mathrm{Co}^{2} \&$ GHG emissions & 1 & 1 & 1 & & & 1 & 2 & 1 & 1 & 3 & 4 & 7 & 4 & 2 & & 28 \\
\hline 2 & Land use exchange & 1 & & & & & 1 & & & & & 1 & 2 & 1 & 1 & & 7 \\
\hline 3 & Resources saving & 1 & & & & & 1 & 1 & 1 & & & 4 & 5 & 3 & 1 & & 17 \\
\hline 4 & Design cost & & & & & & & & 1 & & & 1 & 2 & 3 & & & 7 \\
\hline 5 & Capital cost & 1 & 1 & 1 & & 1 & & & 2 & & 1 & 2 & 6 & 4 & 1 & & 20 \\
\hline 6 & Number of equipment & & & & & & & & & & & & 1 & 2 & 1 & & 4 \\
\hline 7 & Equipment cost & & & 1 & & & & & & & 2 & 2 & 3 & 3 & 2 & & 13 \\
\hline 8 & Labor cost & & 1 & 1 & & 1 & & & 1 & & & 1 & 6 & 5 & 2 & & 18 \\
\hline 9 & Production cost (Operation cost) & 1 & 1 & 1 & & 1 & 1 & 1 & 3 & & 4 & 1 & 9 & 4 & 2 & 1 & 30 \\
\hline 10 & Materials cost & & & 1 & & & 1 & & 2 & & 2 & 3 & 6 & 4 & 2 & 1 & 22 \\
\hline 11 & Transportation cost for feedstock & & & 1 & & & & 1 & 1 & 1 & 3 & 2 & 6 & 4 & 3 & & 22 \\
\hline 12 & Overhead cost & & & 1 & & & & 1 & & & & & 2 & 1 & 1 & & 6 \\
\hline 13 & Response to market \& policy uncertainties & & & & & & & & 1 & & 2 & 4 & 8 & 3 & 2 & & 20 \\
\hline 14 & Storage cost (Feedstock) & & & 1 & & & & 2 & 1 & & 2 & 1 & 5 & 2 & 2 & & 16 \\
\hline 15 & Storage cost (Biofuel) & & & & & & & 2 & 1 & & 1 & 1 & 4 & 1 & 2 & & 12 \\
\hline 16 & Annual investments (Maintenance) & & & 1 & & & & 1 & 1 & & 1 & 2 & 6 & 3 & 1 & & 16 \\
\hline 17 & Life cycle cost with production cost & & & 1 & & & & 1 & & & & 1 & 2 & & & & 5 \\
\hline 18 & Life cycle cost without production & & & & & & & & & & 1 & 1 & 1 & 1 & 1 & & 5 \\
\hline 19 & Feedstock conversion ratio & & & & & & & & & & & & 1 & & & & 1 \\
\hline 20 & Energy saving & & 1 & & & & & 1 & 2 & & & 2 & 5 & 3 & 1 & & 15 \\
\hline 21 & Biomass availability & 1 & & & & & & & 2 & & 1 & 3 & 5 & 3 & 1 & & 16 \\
\hline 22 & Operation efficiency & 1 & & & & & & 1 & 1 & & 2 & 2 & 7 & 2 & 1 & & 17 \\
\hline 23 & Number of offered jobs & & & 1 & & 1 & 1 & 1 & & 1 & & & 6 & 1 & 1 & & 13 \\
\hline 24 & Energy taxes & & & & & & & & & 1 & & & 1 & 2 & & & 4 \\
\hline 25 & Energy policy & & & & & & & & & 1 & & & 3 & 2 & & & 6 \\
\hline 26 & Development status & & & & & & & & & 2 & & & & 1 & & & 3 \\
\hline 27 & Possible subsidies & & & & & & & & & 2 & & & & 1 & & & 3 \\
\hline 28 & Safety & & & & & & 1 & & & & 1 & 1 & & & & & 3 \\
\hline 29 & Public acceptance & & & & & & & & & & & & & 1 & & & 1 \\
\hline 30 & Food prices relation & & & & & & & & & & & & 1 & & & & 1 \\
\hline 31 & Learning curve & & 1 & & & 1 & & & & & & & & & & & 2 \\
\hline & Total observations & 7 & 6 & 12 & 0 & 5 & 7 & 15 & 21 & 9 & 26 & 39 & 110 & 64 & 30 & 2 & 353 \\
\hline
\end{tabular}

From a total of 353 observations noted in the literature from the past two decades, both requirements of capital cost and response to market and policy uncertainties (flexibility) were mentioned 20 times $(5.7 \%)$. A total of $30(8.5 \%)$ observations also stated the production cost requirement. Moreover, both annual investments (maintenance) and biomass availability requirements were mentioned 16 times $(4.5 \%)$ in the previous research on biofuel production. Material cost requirement was observed 22 times $(6.2 \%)$ in the literature reviewed where equipment cost was mentioned 13 times (3.7\%). Finally, energy policy requirement was mentioned 6 times $(1.7 \%)$.

It is important study sustainable strategies to make biofuels a feasible option to increase energy production, as well as national and environmental securities (Awudu \& Zhang, 2012). However, little work has been done to understand

\section{Volume 4 Issue 11, November 2015}




\section{International Journal of Science and Research (IJSR) \\ ISSN (Online): 2319-7064}

Index Copernicus Value (2013): 6.14 | Impact Factor (2014): 5.611

what the optimal size of biomass processing facility itself (Larasati, Liu, \& Epplin, 2012).

High capital cost is one of the major barriers to investing in advanced bio-refineries. Bio-refineries share many similarities with fossil-fuel refineries including the need for significant financing. Fossil-fuel refineries reduce their capital costs through large-scale deployments that take advantage of economies of scale. Crude oil refineries in the United States produce an average of 126,000 barrels per day (bpd), and the largest U.S. refinery can process over 560,000 bpd (3\% of the total U.S. refining capacity) (Wright \& Brown, 2007). A review of other studies shows that bio-refineries capacities are often in the order of $10,000 \mathrm{bpd}$. This is smaller than traditional fossil fuel refineries due in large part to the logistical challenges faced by biomass supply chains that negate savings from the economies of scale (Richard, 2010). Bio-refineries also need to reduce costs even at small-scale but there is no clear path on how to do this.

Industry stakeholders usually support biofuel development when there is an opportunity for profit (Michalopoulos, Landeweerd, Werf-Kulichova, Puylaert, \& Osseweijer, 2011). However, more work is recommended to decide the best unit size of biomass processing (Dwivedi \& Alavalapati, 2009; Larasati, Liu, \& Epplin, 2012; Fawzy \& Componation, 2015), while researchers noticed that the optimal size of a biofuel unit or facility depends on many variables such as capital, operation, transportation, and raw material costs (Wright \& Brown, 2007; Larasati, Liu, \& Epplin, 2012; Fawzy \& Componation, 2015).

The challenge of developing a distributed bio-refinery system is to overcome the conventional cost models that predict the unit cost of a production will decrease as the facility size becomes larger (Arrow, 1962; McDonald \& Schrattenholzer, 2001; Tsuchiya \& Kobayashi, 2004). The concept of "economies of scale" exemplified that there is a positive linear relationship between the size of a facility and the production output. On contrast, the relationship between the size of a facility and the construction, operations, and / or maintenance costs is not linear. The concept does not take into account recent developments in advanced manufacturing technologies. Some of these new manufacturing approaches as well as some new production strategies proposes that bigger may not be better. Big facilities may cost more than smaller ones (Jack, 2009). Some industries, such as aerospace, have begun to explore other design approaches to move away from the bigger is better approach (Componation \& Collopy, 2012). Attention is now being paid to non-technical parameters that can drive costs in developing new systems (Hamaker \& Componation, 2010). In the case of Biofuel Production, the result is complicated because of the transportation costs of distributed and low-density materials. According to Wright \& Brown (2007), there is an optimal plant size for the lowest unit cost of biobased production. Nevertheless, this optimal size is often so large that capital investment for advanced bio-refineries is estimated to be as much as a billion dollars.
BPSs' perspectives and requirements are identified in Fawzy's and Componation study (2015). From that work, ten subject matter experts (SMEs) evaluated a set of eighteen requirements as the most commonly noted requirements for biofuel units manufacturing. From that study, a detailed analysis of the BPSs' requirements is recommended as a future work. In this research, the set of the identified eighteen requirements is used for the stakeholders' requirements assessment for FPUs size selection.

The analysis of three different size FPUs is considered here. First unit's size is selected to be the unit that consumes 2000 tpd. Current petroleum refineries produce more than 10,000 tpd and small corn ethanol plants consume 2,000 tpd of biomass (Wright, Brown, \& Boateng, 2008). This large facility size has been determined to be the optimal size for a Midwestern biorefinery based on typical biomass yields and farm participation. Many biofuel papers use 2000 tpd as their base case size to allow for easy comparison with previous studies even though it is not confirmed as the optimal size (Wright, Brown, \& Boateng, 2008; Anex, et al., 2010; Swanson, Platon, Satrio, \& Brown, 2010; Wright, Daugaard, Satria, \& Brown, 2010; Wright, personal communication, May 14, 2013).

Second unit's size is the unit, which consumes 200, or up to 500 tpd of feedstock, and that is a size that researchers have envisioned that a small group of farmers would invest in (Ringer, Putsche, \& Scahill, 2006; Wright, Brown, \&Boateng, 2008; Wright, Daugaard, Satria, \& Brown, 2010). The capacity and costs for these sizes are comparable to the early ethanol and biodiesel plants (Wright, personal communication, May 14, 2013).

The third unit's size is the smallest unit that consumes 50 tpd of biomass. It is about the largest feasible size for a 'mobile' unit that companies (Dynamotive \& Ensyn) try to produce. In a study done in 2004, it stated, "Fast pyrolysis has proven itself to be a technically viable technology for the 0 to 45 tpd plant size range (LaClaire, Barrett, \& Hall, 2004).

Most of the current research on biofuel production as a renewable energy resource has been done in Europe (Dwivedi \& Alavalapati, 2009). On the other hand, biofuel production has not been as heavily researched as other components of the renewable energy industry in the U.S. Selecting the right size for the biofuel production facility has become more important as production increases in the U.S. In order to design a viable biofuel production system, further analysis is needed. Therefore, this research fills a gap in our knowledge about the optimal size of biofuel production facilities by providing a detailed explanation of the requirements level analysis in addition to the sensitivity analysis of the high-prioritized requirement for this complex decision-making problem.

This research right-sizing FPUs using a Multi Criteria Decision Analysis (MCDA) as a decision-making approach. This will lead to developing a more costeffective production strategy and help prioritize further development efforts. Thus, a general evaluation of the cost- 


\section{International Journal of Science and Research (IJSR) \\ ISSN (Online): 2319-7064}

Index Copernicus Value (2013): 6.14 | Impact Factor (2014): 5.611

effectiveness of modular biofuel production is important considering all requirements and criteria.

\section{Methodology}

The research method was designed to support the goal of enabling further development of an approach for the manufacturing, placement, and optimal sizing of FPUs for biofuel energy production. The focus of this research is to rank stakeholders' requirements based on SME evaluation, to analyze FPUs alternatives to determine how they meet those requirements, and to determine how sensitive the result is to changes in stakeholders' priorities.

The research begins by inquiring about the identified requirements from the previous work on the biofuel manufacturing industry, which focuses on the perspectiveslevel analysis. The model was developed using Logical Decision software V7.2. This software is adopted due to its advanced sensitivity analysis capability. In this model, BPSs' requirements directly connected to the overall goal.

Ten SMEs were interviewed individually to evaluate the eighteen requirements as well as the three FPUs' sizes. These ten SMEs were recommended based on consultation with the management team from the Iowa State University Bioeconomy Institute. The SMEs group for this research included academics researchers and industry representatives all with a minimum of 8 years of experience in the field.

The SMEs evaluated the importance of each of the eighteen requirements using a three-level scale (high importance, medium importance, low importance). The interviewer explained to the SMEs the three levels of evaluation as follows:

- High importance: A small change in this requirement will have a significant measurable impact on the recommended biofuel production strategy.

- Medium importance: A change in this requirement will have a measurable impact on the recommended biofuel production strategy.
- Low importance: A change in this requirement may influence the recommended biofuel production strategy.

Then, the average evaluation is calculated using Equation 1 .

Requirement's weight $=$

$\frac{3 *(\# \text { of } S M E S 1)+2 *(\# \text { of } S M E S 2)+1 *(\# \text { of } S M E S 3)}{\text { Total number of } S M E S}(1)$

Where:

SMEs 1: is the group of those who weighted the requirement's importance as high.

SMEs 2: is the group of those who weighted the requirement's importance as medium.

SMEs 3: is the group of those who weighted the requirement's importance as low.

The ten SMEs evaluated each of the FPUs against each individual requirement using the 2,000 tpd FPU as the base-case since this is the most studied unit size as mentioned in the literature. The SMEs looked at the $200-$ 500 tpd FPU and was asked if it would have an advantage, be the same, or be at a disadvantage in meeting this requirement when compared to the 2,000 tpd FPU base case. A five level scale was used (big advantage advantage - same - disadvantage - big disadvantage). During the data collection, each SME were interviewed individually. In addition, SMEs were able to provide comments and ask the interviewer for some clarification when needed. This process was then completed with the FPUs that consume50 tpd of feedstock.

To determine which FPU provides the best fit for stakeholders' requirements, the raw data collected from the SMEs on evaluation of the requirements using the five level scale was converted to numerical scales as shown in Table 2. This is done to transfer the qualitative evaluation to a quantitative one to have a numeric score representation for each requirement evaluation.

Table 2: Code for Requirements' Evaluation Assessment Scores

\begin{tabular}{|c|c|c|c|c|c|}
\hline The code: & Big advantage & Advantage & Same & Disadvantage & Big disadvantage \\
\cline { 2 - 6 }
\end{tabular}

The raw data on the performance of each FPU against the base case was then calculated by getting the average value of the ten SMEs evaluations for each requirement as shown in Equation 2.

$$
\text { Avg. of requirement's scores }=\frac{\sum_{i=1}^{10} S M E_{i}}{\text { Total number of } S M E S}
$$

After that, each requirement's score at each FPU is calculated by using Equation 3.

Requirement's score $=$

Avg. of requirement's scores * Requirement's weight

Next, the score of each FPU is calculated by using Equation 4.

$$
\text { FPU's score }=\sum_{i=1}^{18}\left(\text { Requirement }_{i} \text { score }\right)
$$

Then, these scores normalized using Equation 5. After that, these normalized data were entered into the developed model using direct entry function in the Logical Decisions V7.2 software.

FPU's score normalization $=$

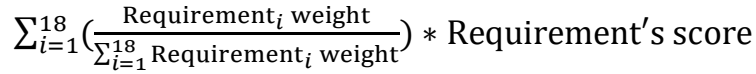

The model was then run and utilities were calculated. To calculate the utility of each alternative, Logical Decisions 


\section{International Journal of Science and Research (IJSR) \\ ISSN (Online): 2319-7064}

Index Copernicus Value (2013): 6.14 | Impact Factor (2014): 5.611

software uses functions called Single-measure Utility Functions (SUFs) which convert measure levels to utilities as explained in the software help. For the three FPUs alternatives, the one with the highest utility would indicate a better fit to stakeholders' requirements and the one with the lowest utility would indicate the poorest fit.

Then, the requirements evaluation by SMEs is used to prioritize the set of identified requirements from high importance levels to the lower importance levels. It is assumed that any requirement had a score of at least 2.5 out of 3 is considered as a high-priority requirement. This means at least 6 out of the ten SMEs evaluated this requirement as a high-priority requirement.

Finally, the sensitivity analysis was conducted on the model using the Logical Decisions software V7.2 (Biggam, 2011). This type of analysis explores the response of the overall utility of alternatives to changes in the relative importance (weight) of each requirement (Biggam, 2011). Logical Decisions software helps to make decisions based on MCDA. In addition to its uses in prior work in the analysis of biofuel stakeholders' perspectives for FPUs alternatives (Fawzy \& Componation, 2015), it has been used in fields such as health and environmental management (Cipollini, Maruyama, \& Zimmerman, 2005; Moffett, Dyer, \& Sarkar, 2006, Honoré, Fos, Smith, Riley, \& Kramarz, 2010). This software allows evaluatingalternative solutions by considering multibal requirments simultaneously, which simplifies thedecision-making proces with logical illustration.

\section{Results}

The analysis investigated both the results of the MCDA model and the comments from the SMEs. The smallest FPU's size, with a capacity of 50 tpd, is the best option based on the study that identified the five perspectives. This research used the eighteen stakeholders' requirements identified in the previous research (Fawzy \& Componation, $2015)$ to create the model. As mentioned in the methodology, all requirements in the model are connected directly to the overall goal, without the clustering on the sub-objectives. Then, all the weights were assigned by using the direct entry function in the software. Moreover, Logical Decisions V7.2 is used in this analysis. Figure 1 presents the final hierarchy with assigned weights.

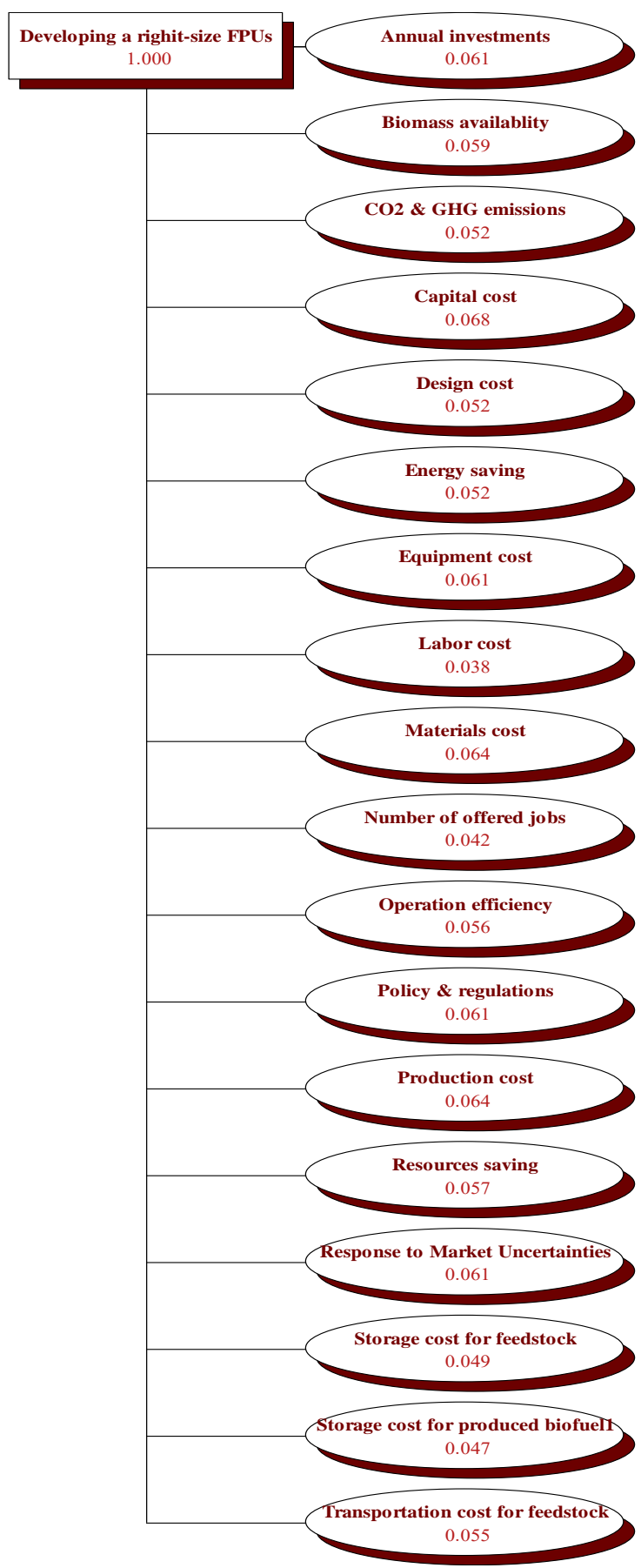

Figure 1: Final Hierarchy with Assigned Weights Using Logical Decisions V7.2

After analyzing the model shown in Figure 1, FPU with a capacity of 50 tpd was found as the best selection with a utility equal to 0.523 . This result coincided with the result of the previous study which conducted on the perspectiveslevel (Fawzy \& Componation, 2015). Similarly, FPU with a capacity between 200 - 500 tpd became the second best option with a utility equal to 0.519 . Finally, FPU with a capacity of at least 2000 tpd was found as the least selection with a utility equal to 0.500 . Figure 2 presents the three alternatives ranking and utilities for the model based on the stakeholders' requirements analysis. 


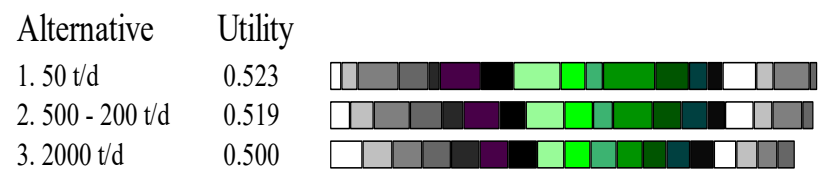

$\begin{array}{lll}\square \text { Capital cost } & \square \text { Production cost } & \square \text { Materials cost } \\ \square \text { Annual investments } & \square \text { Equipment cost } & \square \text { Response to market uncertainties } \\ \square \text { Policy \& regulations } & \square \text { Biomass availablity } & \square \text { Resources saving } \\ \square \text { Operation efficiency } & \square \text { Transportation cost for feedstock } & \square \text { Design cost } \\ \square \text { CO }^{2} \text { \& GHG emissions } & \square \text { Energy saving } & \square \text { Storage cost for feedstock } \\ \square \text { Storage cost for produced biofuel } & \square \text { Number of offered jobs } & \square \text { Labor cost }\end{array}$

Figure 2: Final Utilities for the Alternatives Analysis from the Logical Decisions Model

Preference Set $=$ NEW PREF. SET

It is noteworthy to mention that the equipment cost requirement is considered as the cost of the first group of equipment to run the facility plus any replacement or upgrades during the facility lifetime. The requirement of response to market and policy uncertainties means that the unit's flexibility and its ability to respond to demand and other market and political changes. Furthermore, the annual investment requirement represents the maintenance cost and the investors' ability to invest in such process annually. As stated by a SME, maintenance cost on average equals to $5 \%$ of overall cost, even the SMEs evaluated this requirement as a high-prioritized one. Incidentally, according to some SMEs, transportation cost represent approximately $10 \%$ of the total cost, storages cost for raw material is around $22 \%$ and for final product it is represents another $10 \%$ to $17 \%$ of the total cost.

Two of the SMEs from the industry sector believe that the medium size facility is the best alternative and the smallest FPUs will lose the advantages of scale. Moreover, one of those two SMEs stated that this is not a linear decision. Instead of considering which size fit the stakeholders' requirements best, stakeholders should decide to start manufacturing the smallest size then duplicate it or produce bigger ones as needed. In other words, the first unit serves as an experimental unit to examine the situation and improve the learning curve.

For this research, the model is created using the Logical Decisions software V7.2 by considering all the eighteen requirements and their weights evaluated by the ten SMEs. The weights given to the requirements in the software using the direct enter method. As a result the small FPU has the highest utility comparing to the other alternatives. Thus, it is selected as the best alternative according to the stakeholders' requirements and the requirements evaluation of the three alternatives.

Figure 3 shows the results of more investigations on the requirements and their priorities. The eighteen requirements ranking from the highest importance to the lowest one are shown. According to the SMEs' evaluations, eight requirements had a score of at least 2.5 out of 3 , which is considered a high-priority requirement. Thus, eight out of eighteen requirements get high-prioritized importance based on the ten SMEs evaluation. As shown in Figure 3, the capital cost requirement is evaluated as the highest important requirement for the decision-making with score of 2.9 out of 3 . This means that this requirement has the highest influence on the decision were nine out of the ten SMEs ranked it with high importance evaluation. Following the capital cost, both requirements of materials cost (feedstock cost) and production cost got the score equal to 2.7 out of 3 . Then, four of the eighteens evaluated requirements got a score equal to 2.6 out of 3 . These four requirements are: Policy $\&$ regulations requirement, annual investments (maintenance) requirement, the unit's response to market \& policy uncertainties requirement, and equipment cost requirement. Finally, the biomass availability requirement, which is based on the current situation, is considered as a high-priority requirement with score equal to 2.5 out of 3 , which represent the evaluation of 6 out of 10 SMEs. These high-priority requirements are the ones that are examined in the sensitivity analysis. 


\section{International Journal of Science and Research (IJSR) \\ ISSN (Online): 2319-7064}

Index Copernicus Value (2013): 6.14 | Impact Factor (2014): 5.611

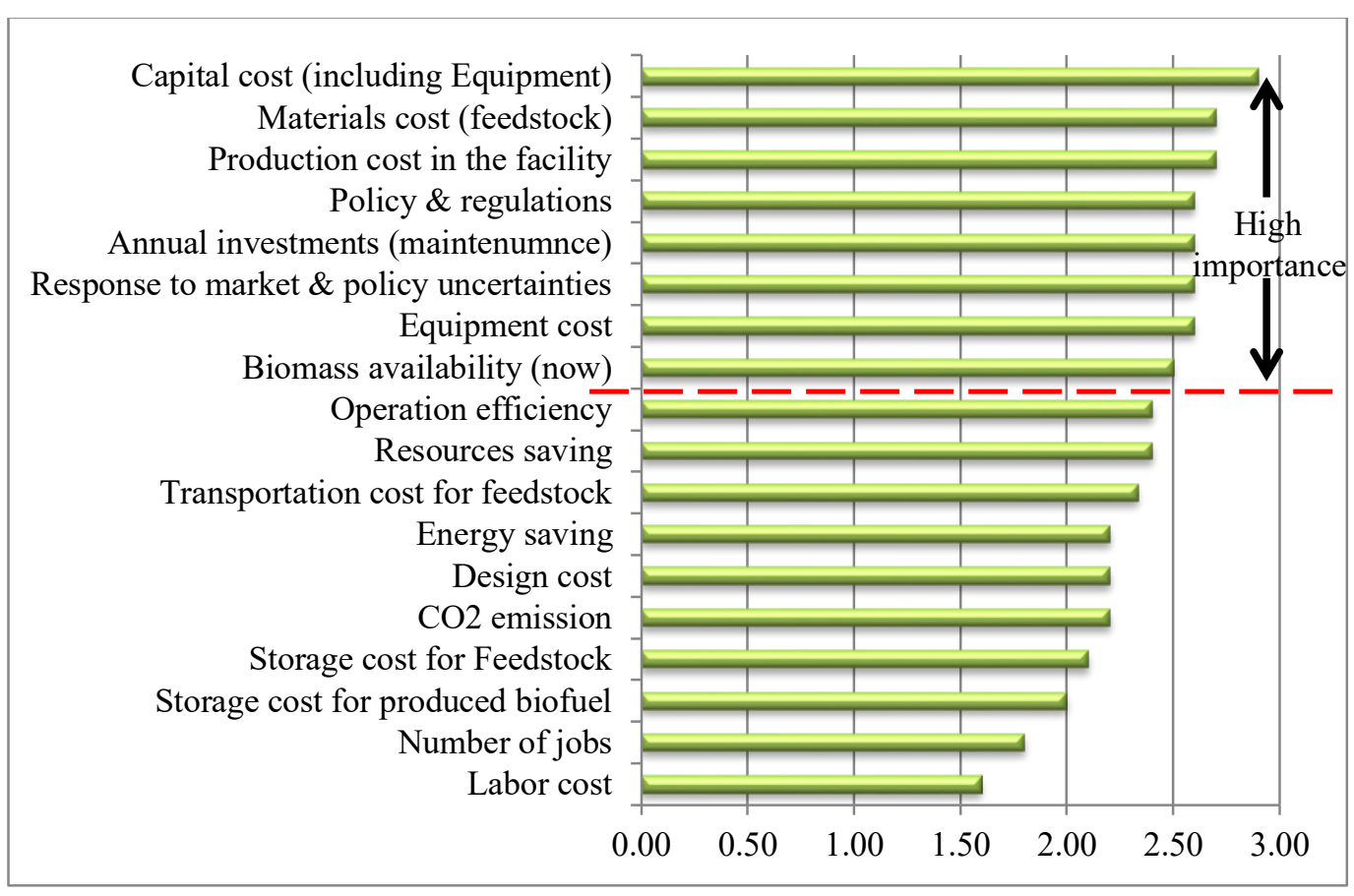

Figure 3: Ranked Requirements of FPUs' Manufacturing

\section{Sensitivity Analysis}

To study the result robustness, sensitivity analysis is conducted. Sensitivity analysis on the requirements level helps to understand which requirement(s) affecting the decision exactly under the general perspectives. In addition, to know under which condition(s) the decision will change. Moreover, what will be the best alternative for other situations, if exist.

A sensitivity analysis is applied on the requirements using Logical Decisions V7.2. As an output from this research, eight out of the eighteen requirements were ranked as highprioritized requirements according to the SMEs evaluation. Since the sensitivity analysis in this research is focused more on the high-prioritized requirements, it was done on these eight high-prioritized requirements. When a sensitivity analysis was performed on each requirement of these eight, the weight given to that requirement is adjusted up or down to examine when the utilities ranking will change. On the other hand, the weights of to the other seventeen requirements in the model are adjusted proportionally. Since the capital cost has the highest score in the requirements importance evaluation by SMEs, a sensitivity analysis was conducted on this requirement first (Figure 4). The weight of the capital cost requirement was varied from 0 to $100 \%$. The sensitivity analysis showed that when the capital cost requirement was weighted at any value from 0 to $11 \%$ the smallest FPUs (50 tpd) was recommended. When the weight given to the capital cost requirement was increased to $12 \%$, the medium sized FPUs (200 to 500 tpd) became the recommended size. When weight of the capital cost requirement exceeds $16 \%$, the big sized FPUs (at least 2000 tpd) were recommended. The vertical line at weight of $7 \%$ was the weight given to the capital cost requirement in the initial analysis and at this point, the smallest FPUs is the recommended unit.
Nine out of the ten SMEs believe that this requirement is the most important one for any kind of manufacturing. Moreover, some SMEs mentioned that this is the reason behind the focus of previous research on this requirement more than other ones.

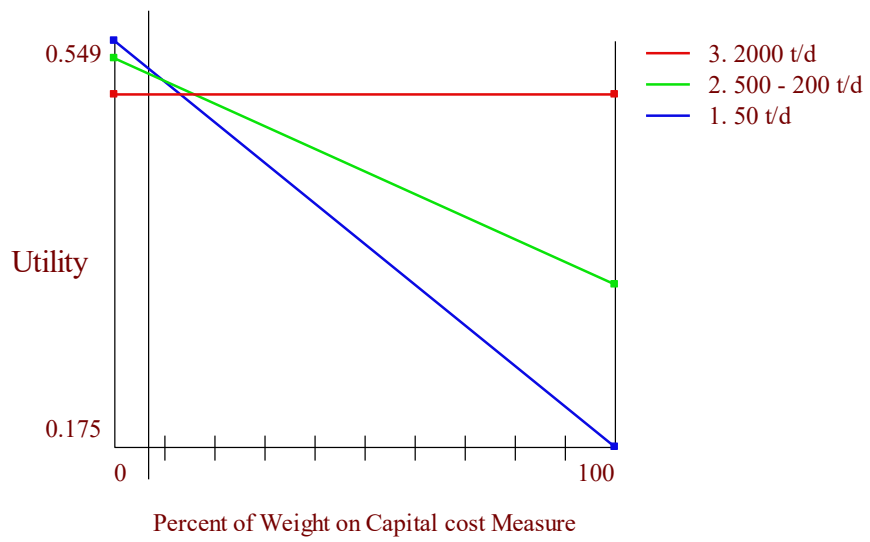

Figure 4: Sensitivity Analysis on Capital Cost Requirement

The same analysis was conducted on each one of the other high-prioritized requirements on sequence (Figure 5). This is done to examine the decision robustness. It is interesting to note that the smallest FPU is recommended in approximately $43(53.75 \%)$ of the 80 sensitivity analysis cases. When policy and regulations, raw material cost, or flexibility requirements were weighted no more than $4 \%$, the small FPU was recommended. An increase in the weights of the other requirements such as: production cost or equipment cost requirements resulted in the big FPUs becoming the recommended alternative when the weight increases by approximately $20 \%$ or more. For the biomass availability requirement, based on the current situation, as shown in Figure 5, the 50-tpd units is the best alternative when the importance of this requirement becomes more than 5\%. This result matches the first impression of most of the SMEs, where they believe that the smallest FPUs will have the 


\section{International Journal of Science and Research (IJSR) \\ ISSN (Online): 2319-7064 \\ Index Copernicus Value (2013): 6.14 | Impact Factor (2014): 5.611}

advantages to be located wherever the feedstock is available. This is one of the strongest advantages of having such a distributed system. A summary of all requirements' weights and their impacts on the recommended alternative are shown in Figure 5.

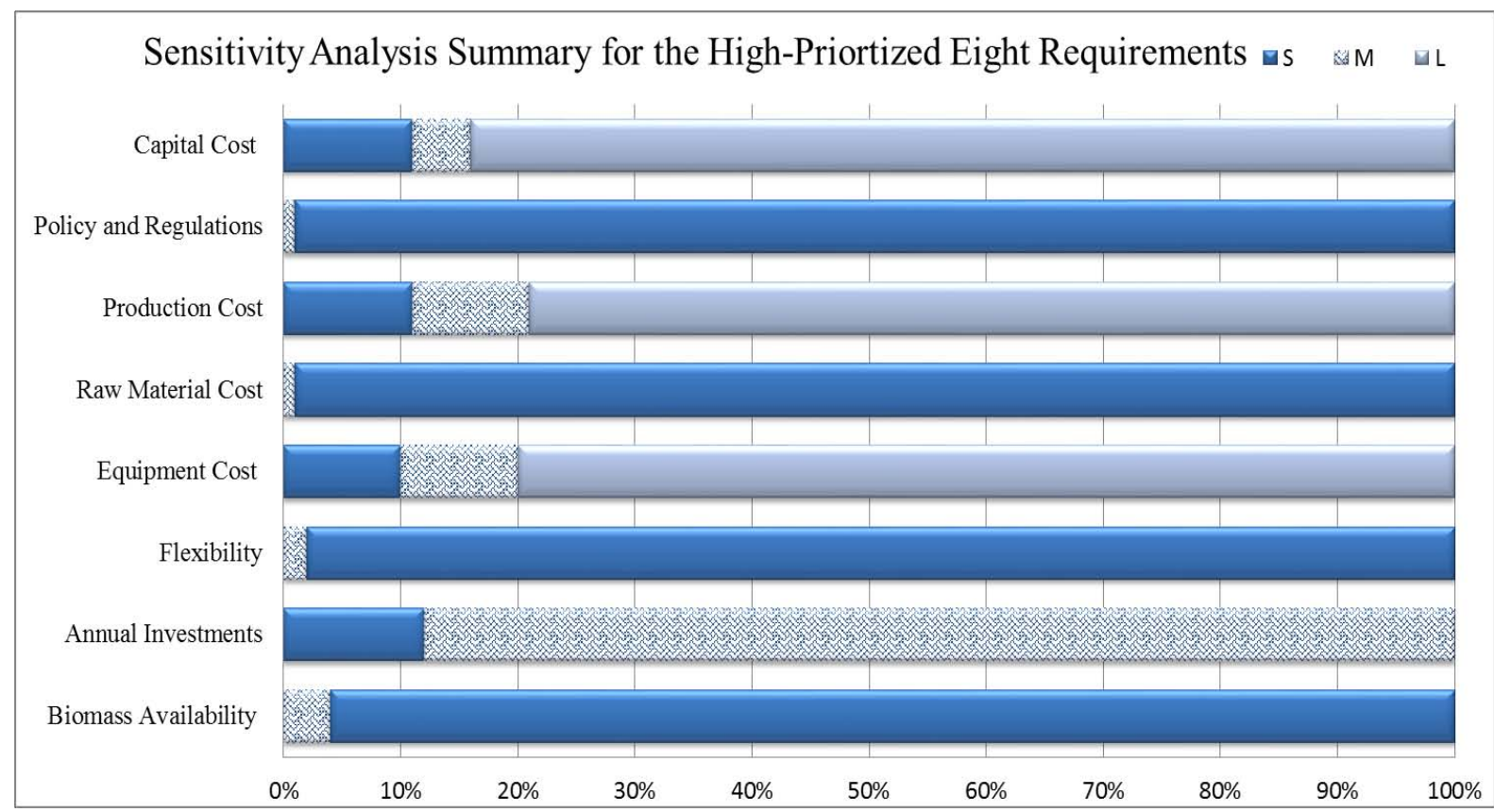

Figure 5: Sensitivity Analysis Summary for the High-Prioritized Eight Requirements

It is also worthy to note that some SMEs mentioned that the production cost in the facility is also one of the most important requirements affecting decision after the capital cost requirement. Seven out of the ten SMEs believe in that the raw materials cost (feedstock cost) requirement is one of the factors that has a significant impact on the decisionmaking process, because there is a strong positive relation between this type of cost and the final product price. On the other hand, other SMEs believe that this requirement is not that important due to the units' flexibility advantage in addition to the availability of this raw material for biofuel production. This requirement shows the impact of the input side of the operation on the decision-making process. However, if this requirement has no weight value, the medium and smallest FPUs alternatives will have the same utilities in the sensitivity analysis.
Due to the convergence of utilities between the smallest units' size alternative and the medium units' size alternative, the relationship between these two alternatives was studied further. Figure 1shows that overall the smaller unit's size of FPUs (50 tpd) has a slightly better advantage than the medium FPUs size $(500-200$ tpd $)$. More specifically, it shows that the medium size of FPUs, due to its distribution, is worst in the transportation cost of raw material, biomass availability, and number of jobs offered by the facility. Moreover, the advantages of requirements such as material cost, raw material storages cost, response to market and policy changes is minimal. Figure 6illustrates all the advantages and disadvantages on the requirements level base for both small and medium FPUs' sizes using tornado chart. 
International Journal of Science and Research (IJSR)

ISSN (Online): 2319-7064

Index Copernicus Value (2013): 6.14 | Impact Factor (2014): 5.611

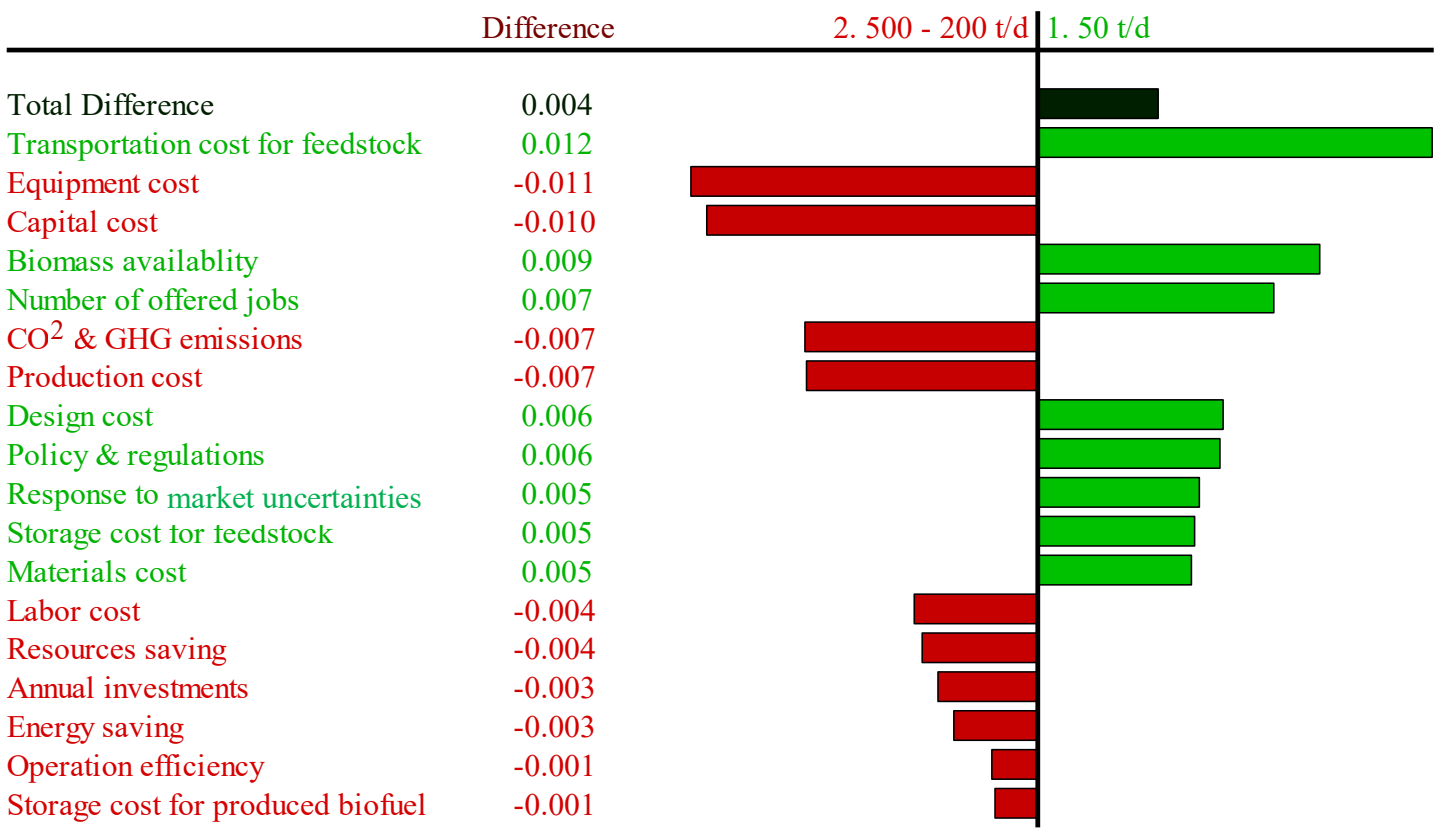

Figure 6: Sensitivity Analysis on Best Two FPUs' Sizes

Preference Set $=$ NEW PREF. SET

An industrial SME mentioned that some of the requirements could depend on others. He argued that equipment costs could be part of the capital cost and labor cost could be part of the production cost. As a part of the sensitivity analysis, the authors decided to remove these two requirements which could be related to capital and operation costs and rerun the model to see the impact of this action. As shown in Table 3,

the smallest units' size got greater value $(0.2313$ out of 1$)$ than what it had with the existence of the two removed requirements (0.0934). In this case, removing these requirements did not affect the final decision. In fact, it did support the decision.

Table 3: Scores for the Alternatives Analysis under SME's Assumptions

\begin{tabular}{|c|c|c|c|c|c|c|c|c|c|c|c|c|c|c|c|}
\hline \multirow[t]{2}{*}{ Requirements } & \multicolumn{4}{|c|}{ Priorities } & \multirow{2}{*}{ 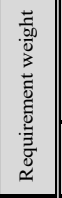 } & \multicolumn{3}{|c|}{$\begin{array}{c}\text { Alternatives (Facility size) } \\
\text { evaluations average } \\
\text { Pugh Concept - feedstock inputs tpd }\end{array}$} & \multicolumn{3}{|c|}{$\begin{array}{c}\text { Alternatives (Facility size) } \\
\text { The weighted Scores } \\
\text { Pugh Concept - feedstock inputs tpd }\end{array}$} & \multirow{2}{*}{ 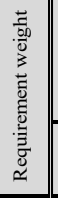 } & \multicolumn{3}{|c|}{$\begin{array}{c}\text { Alternatives (Facility size) } \\
\text { The weighted Scores } \\
\text { Pugh Concept - feedstock inputs tpd }\end{array}$} \\
\hline & $\mathrm{H}$ & M & $\mathrm{L}$ & \begin{tabular}{|c||} 
no \\
answer
\end{tabular} & & $\begin{array}{c}\geq 2000 \text { tpd } \\
\text { (base) }\end{array}$ & $\begin{array}{c}200-500 \\
\text { tpd }\end{array}$ & $\leq 50 \mathrm{tpd}$ & $\begin{array}{c}\geq 2000 \text { tpd } \\
\text { (base) }\end{array}$ & $\begin{array}{c}200-500 \\
\text { tpd }\end{array}$ & $\leq 50 \mathrm{tpd}$ & & $\begin{array}{c}\geq 2000 \text { tpd } \\
\text { (base) }\end{array}$ & $\begin{array}{c}200-500 \\
\text { tpd }\end{array}$ & $\leq 50 \mathrm{tpd}$ \\
\hline $\mathrm{CO}^{2}$ emission & 4 & 4 & 2 & 0 & 2.20 & 0 & 0.1111 & -0.4444 & 0 & 0.2444 & -0.9778 & 0.06 & 0 & 0.0064 & -0.0256 \\
\hline Resources saving & 5 & 4 & 1 & 0 & 2.40 & 0 & 0.1250 & -0.1250 & 0 & 0.3000 & -0.3000 & 0.06 & 0 & 0.0078 & -0.0078 \\
\hline Design cost & 4 & 4 & 2 & 0 & 2.20 & 0 & 0.3333 & 0.7778 & 0 & 0.7333 & 1.7111 & 0.06 & 0 & 0.0192 & 0.0448 \\
\hline Capital cost (including Equipment) & 9 & 1 & 0 & 0 & 2.90 & 0 & -0.7000 & -1.3000 & 0 & -2.0300 & -3.7700 & 0.08 & 0 & -0.0531 & -0.0986 \\
\hline Production cost in the facility & 7 & 3 & 0 & 0 & 2.70 & 0 & -0.4444 & -0.8889 & 0 & -1.2000 & -2.4000 & 0.07 & 0 & -0.0314 & -0.0628 \\
\hline Materials cost (feedstock) & 7 & 3 & 0 & 0 & 2.70 & 0 & 0.4000 & 0.7000 & 0 & 1.0800 & 1.8900 & 0.07 & 0 & 0.0282 & 0.0494 \\
\hline Transportation cost for feedstock & 5 & 2 & 2 & 1 & 2.33 & 0 & 1.1111 & 2.0000 & 0 & 2.5926 & 4.6667 & 0.06 & 0 & 0.0678 & 0.1221 \\
\hline Response to market \& policy uncertainties & 7 & 2 & 1 & 0 & 2.60 & 0 & 0.5556 & 0.8889 & 0 & 1.4444 & 2.3111 & 0.07 & 0 & 0.0378 & 0.0604 \\
\hline Storage cost for feedstock & 3 & 5 & 2 & 0 & 2.10 & 0 & 0.5000 & 0.9000 & 0 & 1.0500 & 1.8900 & 0.05 & 0 & 0.0275 & 0.0494 \\
\hline Storage cost for produced biofuel & 4 & 1 & 4 & 1 & 2.00 & 0 & -0.2222 & -0.3333 & 0 & -0.4444 & -0.6667 & 0.05 & 0 & -0.0116 & -0.0174 \\
\hline Annual investments (maintenumnce) & 6 & 4 & 0 & 0 & 2.60 & 0 & 0.3000 & 0.1000 & 0 & 0.7800 & 0.2600 & 0.07 & 0 & 0.0204 & 0.0068 \\
\hline Energy saving & 3 & 6 & 1 & 0 & 2.20 & 0 & -0.5000 & -0.7000 & 0 & -1.1000 & -1.5400 & 0.06 & 0 & -0.0288 & -0.0403 \\
\hline Biomass availability (now) & 6 & 3 & 1 & 0 & 2.50 & 0 & 0.8000 & 1.4000 & 0 & 2.0000 & 3.5000 & 0.07 & 0 & 0.0523 & 0.0915 \\
\hline Operation efficiency & 4 & 6 & 0 & 0 & 2.40 & 0 & -0.5000 & -0.6000 & 0 & -1.2000 & -1.4400 & 0.06 & 0 & -0.0314 & -0.0377 \\
\hline Policy \& regulations & 6 & 4 & 0 & 0 & 2.60 & 0 & -0.1250 & 0.2500 & 0 & -0.3250 & 0.6500 & 0.07 & 0 & -0.0085 & 0.0170 \\
\hline \multirow[t]{2}{*}{ Number of jobs } & 2 & 4 & 4 & 0 & 1.80 & 0 & 1.0000 & 1.7000 & 0 & 1.8000 & 3.0600 & 0.05 & 0 & 0.0471 & 0.0800 \\
\hline & & & & & & & \multicolumn{2}{|c|}{ FINAL SCORS: } & 0 & 5.7254 & 88444 & 1 & 0 & 0.1497 & 0.2313 \\
\hline
\end{tabular}

For this research, three types of sensitivity analysis are conducted. First sensitivity analysis is done to examine the result robustness. This sensitivity analysis is done by changing each requirement weight from $0 \%$ (no importance give to the requirement) to $100 \%$ (all the importance give to the requirement). Then, authors observe the effect of these 


\section{International Journal of Science and Research (IJSR) \\ ISSN (Online): 2319-7064}

Index Copernicus Value (2013): 6.14 | Impact Factor (2014): 5.611

changes on the result. From this analysis authors found that small FPU size has the advantage in four out of the eight high-prioritized requirements. Second sensitivity analysis is done to examine differences between the best two alternatives, which are the small and medium FPUs sizes. This type of sensitivity analysis is done using a tornado chart to illustrate the advantages and disadvantages of each of the two FPUs sizes comparing to each other based on the requirements-level. As a result for this analysis, the small FPU size got the advantage at eight out of the eighteen requirements comparing to the medium FPU size. Last sensitivity analysis is done to investigate the dependency of two of the requirements according to an industrial SME argument. This type of sensitivity analysis is done by using a Microsoft Excel model by excluding the two requirements. As a result of this sensitivity analysis, authors found that the smallest FPU size got the highest score again comparing to the other two alternatives.

\section{Conclusion}

This research supports the decision-making process by providing insights on how stakeholder requirements influence selection of the appropriate facility size for biofuel production. From requirements-level, the smallest FPUs' size is the best option for the eighteen requirements, but under specific conditions. From the investigation on the requirements-level the authors found that eight out of the identified eighteen requirements as were ranked as highprioritized requirements according to the SMEs evaluation. Those eight high-prioritized requirements consecutively are: Capital cost, raw materials cost, production cost, policy and regulations, annual investments, response to market and policy uncertainties and changes, equipment cost, and biomass availability.

\section{Recommendations}

Three out of the eight highly important requirements present the big FPUs alternative as the best alternative under a wide range of weights. In other words, for capital, production, and equipment costs, the big FPUs alternative is recommended whenever the weight of each of these requirements exceed one fifth of the total requirements weights. From this research, it is recommended that more work is needed to reduce capital, production, and equipment costs for FPUs manufacturing. This will reduce these costs variation among the three alternate FPUs sizes. Using appropriate application of advanced manufacturing technologies is one approach, since the study of advanced manufacturing technologies and its relationship with business' strategy is currently receiving significant scholarly attention (Kotha \& Swamidass, 2000). In addition, thinking small in design and manufacturing perceptions is another approach that could be followed (Dahlgren, Göçmen, Lackner, \& van Ryzin, 2013). The combination of these two approaches could also be effective in reducing capital, production, and equipment costs.

Four out of the eight high-prioritized requirements presented the small FPUs size as the best option whenever each requirement weight exceeded the $5 \%$. Overall, the medium FPUs in the analysis became the second best alternative with a close utility to the first alternative. However, only one requirement of the eight high-prioritized requirements presented the medium unit's size as the best option for FPU manufacturing with a wide range of weights. This requirement is the annual investment requirement including the maintenance cost of the facility. This helps to think about the available advantages of maintenance cost for the medium unit's size and how it could be implemented in the smallest unit's size by encouraging engineers to think small in design and manufacturing perceptions as Dahlgren et al. (2013) recommended.

Authors believe this research supports the idea that "bigger is not always better." This research highlights some requirements' impacts on the decision. Moreover, it paved a way to select the unit's size for biofuel production based on a scientific methodology for the decision-making considering stakeholders' requirements.

Even though the smallest FPUs size is the best units' size to pass both analysis levels of perspectives, from Fawzy and Componation's study (2015), and requirements, from the current research, the biggest FPUs size is recommended as the second best alternative in $37.5 \%$ ( 3 out of 8 requirements) of the high-prioritized requirements, from the current research. On the other hand, the medium FPUs size is recommended as the second best alternative based on the perspectives'-level analysis as shown in Fawzy and Componation (2015). This thorough analysis of the requirements-level provides a clear picture on what engineers should focus on if they want to think small in design and manufacturing perceptions. Moreover, this work illustrates the effect of each of the top eight requirements on the FPUs production decision and which of them could be improved to reach smaller verses bigger FPUs.

\section{References}

[1] Anex, R. P., Aden, A., Kazi, F. K., Fortman, J., Swanson, R. M., Wright, M. M., . . Dutta, A. (2010). Techno-economic comparison of biomass-totransportation fuels via pyrolysis, gasification, and biochemical pathways. Fuel, 89, pp. 29-35.

[2] Arrow, K. J. (1962). The Economic Implications of Learning by Doing. The Review of Economic Studies, 29(3), pp. 155-173.

[3] Awudu, I., \& Zhang, J. (2012). Uncertainties and Sustainability Concepts in Biofuel Supply Chain Management: A Review. Renewable and Sustainable Energy Reviews, 16(2), pp. 1359-1368.

[4] Biggam, J. (2011). Succeeding With Your Master'S Dissertation: A Step-By-Step Handbook.New York City, New York, U.S: McGraw-Hill International.

[5] Cipollini, K. A., Maruyama, A. L., \& Zimmerman, C. L. (2005). Planning for Restoration: A Decision Analysis Approach to Prioritization. Restoration Ecology Journal, 13(3), pp. 460-470.

[6] Componation, P. J., \& Collopy, P. D. (2012). Systems Engineering Theory: Addressing Complexity and Uncertainty in Space Systems Architecture. AIAA Space 2012 Conference \& Exposition. Pasadena, CA, USA.

\section{Volume 4 Issue 11, November 2015}




\section{International Journal of Science and Research (IJSR) \\ ISSN (Online): 2319-7064 \\ Index Copernicus Value (2013): 6.14 | Impact Factor (2014): 5.611}

[7] Dahlgren, E., Göçmen, C., Lackner, K., \& van Ryzin, G. (2013). Small modular infrastructure. The Engineering Economist, 58(4), pp. 231-264.

[8] Dwivedi, P., \&Alavalapati, \&. J. (2009). Stakeholders' perceptions on forest biomass-based bioenergy development in the southern US. Energy Policy, 37(5), pp. 1999-2007.

[9] Dyer, J. (2005). MAUT - Multiattribute Utility Theory. In J. Figueira, S. Greco, \& M. Ehrogott, Multiple Criteria Decision Analysis: State of the Art Surveys, 78, pp. 265292. New York City, New York, U.S: Springer New York.

[10] EIA. (2001). Monthly Energy Review. (The U.S. Energy Information Administration) Retrieved December 03, 2013, from Table 1.3 and 10.1: http://www.eia.gov/energyexplained/index.cfm?page=ren ewable home

[11] EIA. (2014). Monthly Energy Review. (The U.S. Energy Information Administration) Retrieved December 01, 2014, from Table 1.3 and 10.1: http://www.eia.gov/energyexplained/index.cfm?page=ren ewable home

[12] Fawzy, M., \& Componation, P. (2014). Biofuel Production: Stakeholders' Identification. Journal of Management and Engineering Integration, 7(1), pp. 1522.

[13] Fawzy, M., \& Componation, P. (2015). Biofuel Production: Utilizing Stakeholders' Perspectives for Units Manufacturing. Engineering Management Journal,27(2), pp. 85-96.

[14] Freeman, R. E. (1984). Strategic management: A stakeholder approach. Cambridge University Press.

[15] Hamaker, J. W., \& Componation, P. J. (2010). Using our Right Brains to Improve Space Project Cost Estimating. Journal of Cost Analysis and Parametrics, 1(2), pp. 5975.

[16] Honoré, P. A., Fos, P. J., Smith, T., Riley, M., \& Kramarz, K. (2010). Decision Science: A Scientific Approach to Enhance Public Health Budgeting. Journal of Public Health Management and Practice, 16(2), pp. 98-103.

[17] Jack, M. W. (2009). Scaling laws and technology development strategies for biorefineries and bioenergy plants. Bioresource technology, 100(24), pp. 6324-6330.

[18] Kotha, S., \&Swamidass, P. (2000). Strategy, advanced manufacturing technology and performance: empirical evidence from US manufacturing firms. Journal of Operations Management, 18(3), pp. 257-277.

[19] LaClaire, C. E., Barrett, C. J., \& Hall, K. (2004). Technical, environmental and economic feasibility of biooil in New Hampshire's north country. Durham, NH: University of New Hampshire.

[20] Larasati, A., Liu, T., \&Epplin, F. M. (2012, December). An Analysis of Logistic Costs to Determine Optimal Size of a Biofuel Refinery. Engineering Management Journal, 24(4), pp. 63-72.

[21] McDonald, A., \&Schrattenholzer, L. (2001). Learning Rates for Energy Technologies. Energy Policy, 29(4), pp. 255-261.

[22] Michalopoulos, A., Landeweerd, L., Werf-Kulichova, Z. V., Puylaert, P. G., \& Osseweijer, P. (2011). Contrasts and synergies in different biofuel reports.Interface Focus, 1(2), pp. 248-254.

[23] Min, H. (1994). International supplier selection: A multiattribute utility approach. International Journal of
Physical Distribution \& Logistics Management, 24(5), pp. 24-33.

[24] Moffett, A., Dyer, J. S., \& Sarkar, S. (2006). Integrating biodiversity representation with multiple criteria in NorthCentral Namibia using non-dominated alternatives and a modified analytic hierarchy process. Biological Conservation, 129(2), pp. 181-191.

[25] Richard, T. L. (2010). Challenges in scaling up biofuels infrastructure. Science, 329(5993), pp. 793-796.

[26] Ringer, M., Putsche, V., \& Scahill, J. (2006). Large-scale pyrolysis oil production: a technology assessment and economic analysis. Colorado: National Renewable Energy Laboratory.

[27] Swanson, R. M., Platon, A., Satrio, J. A., \& Brown, R. C. (2010). Techno-economic analysis of biomass-to-liquids production based on gasification. Fuel, 89, pp. 11-19.

[28] Tsuchiya, H., \& Kobayashi, O. (2004). Mass Production Cost of Pem Fuel Cells by Learning Curve. International Journal of Hydrogen Energy, 29(10), pp. 285-900.

[29] Wright, M. M., Brown, R. C., \&Boateng, A. A. (2008). Distributed processing of biomass to bio-oil for subsequent production of Fischer-Tropsch liquids Biofuels, Bioproducts and Biorefining, 2(3), pp. 229-239.

[30] Wright, M. M., Daugaard, D. E., Satria, J. A., \& Brown, R. C. (2010). Techno-economic Analysis of Biomass Fast Pyrolysis to Transportation Fuels. Fuel, 89(1), pp. 2-10.

[31] Wright, M., \& Brown, R. C. (2007). Establishing the optimal sizes of different kinds of biorefineries. Biofuels, Bioproducts and Biorefining, 1(3), pp. 191-200.

\section{Author Profile}

Dr. Mostafa F. Fawzy is a researcher working on decision-making research for complex problems. He received his Ph.D. in Industrial Engineering at Iowa State University. $\mathrm{He}$ is interested in the development of decision-making support systems for complex problems such as energy manufacturing. Moreover, his research interests include lean principles applications, strategic planning, and performance enhancement. Dr. Fawzy worked in the consulting field as a development engineer and project manager for five years in the Jeddah Municipality and two consulting offices.

Dr. Paul J. Componation is Professor and Chair of the Industrial, Manufacturing, \& Systems Engineering Department at the University of Texas at Arlington. His research interests are in development and optimization of complex systems in aerospace, transportation, and energy. Prior to his current position, Dr Componation served as the Director of Graduate Education for Engineering Management at Iowa State University and the Systems Engineering Program Coordinator at The University of Alabama in Huntsville.

Dr. Guiping $\mathrm{Hu}$ is an Assistant Professor of Industrial and Manufacturing Systems Engineering and an affiliated faculty of the Bioeconomy Institute at Iowa State University. Her research interests include operations research and optimization with applications on techno-economic analysis, bioenergy pathways and supply chain management. 